\title{
Multicenter phase II study of apatinib treatment for metastatic gastric cancer after failure of second-line chemotherapy
}

\author{
Hanguang Ruan ${ }^{1,2}$, Junlin Dong ${ }^{3}$, Xueliang Zhou ${ }^{4}$, Juan Xiong ${ }^{5}$, Hua Wang ${ }^{5}$, \\ Xiaoming Zhong ${ }^{5}$ and Xiaolong $\mathrm{CaO}^{6}$ \\ ${ }^{1}$ Department of Oncology, The Third Hospital of Nanchang City, Jiangxi Province, Nanchang, China \\ ${ }^{2}$ Graduate School of Jiangxi Medical College, Nanchang University, Nanchang, China \\ ${ }^{3}$ Department of Oncology, Zhuhai Peoples' Hospital of Guang Dong Province, Zhu Hai, China \\ ${ }^{4}$ Cancer Centre, The First Affiliated Hospital of Nanchang University, Nanchang, China \\ ${ }^{5}$ Department of Cancer Radiotherapy, Jiangxi Cancer Hospital of Jiangxi Province, Nanchang, China \\ ${ }^{6}$ Department of Oncology, Panyu District Center Hospital of Guang Dong Province, Guangzhou, China \\ Correspondence to: Xiaolong Cao, email: jxcancer@163.com \\ Keywords: Apatinib, metastatic gastric cancer (mGC), effective, adverse events \\ Received: July 05, $2017 \quad$ Accepted: August 17, $2017 \quad$ Published: September 19, 2017
}

Copyright: Ruan et al. This is an open-access article distributed under the terms of the Creative Commons Attribution License 3.0 (CC BY 3.0), which permits unrestricted use, distribution, and reproduction in any medium, provided the original author and source are credited.

\section{ABSTRACT}

Apatinib is a tyrosine kinase inhibitor and vascular endothelial growth factor receptor 2 (VEGFR-2) targeted drug. A phase I clinical trial showed that this agent has antitumor activity in Chinese patients with metastatic gastric cancer (mGC). The aim of this study was to investigate the safety and efficacy of apatinib treatment in patients with mGC.

This was an open-label, multicenter, single-arm study involving four institutions in China. We enrolled 42 patients from March 2015 to October 2015 who experienced tumor progression after second-line chemotherapy and had no other treatment options that clearly conferred a survival benefit. Oral apatinib ( $850 \mathrm{mg}$ daily) was administered within $\mathbf{3 0}$ min of eating breakfast, lunch, or dinner on days 1 through 28 of each 4-week cycle.

The median progression-free survival (PFS) time and median overall survival (OS) time were 4.0 months (95\% CI, 2.85-5.15) and 4.50 months (95\% CI, 4.034.97), respectively. The disease control rate (DCR) and objective response rate (ORR) were, respectively, $78.57 \%$ and $9.52 \%$ after 2 cycles and $57.14 \%$ and $19.05 \%$ after 4 cycles. The main adverse events (AEs) were secondary hypertension, elevated aminotransferase, and hand-foot syndrome, with incidences of $35.71 \%, 45.24 \%$, and $40.48 \%$, respectively. The most common grade 3 to 4 AEs were secondary hypertension and elevated aminotransferase, with incidences of $7.14 \%$ each.

Apatinib is effective and safe in heavily pretreated patients with mGC who fail to respond to two or more prior chemotherapy regimens. Toxicities were tolerable or could be clinically managed.

\section{INTRODUCTION}

Gastric cancer is a common malignant tumor that is associated with a very high incidence and mortality rate in our country. Its rates of morbidity and mortality are higher in rural areas than in the city. Locally advanced or metastatic gastric cancer has been diagnosed in the vast majority of patients by the time they enter the hospital
[1-3]. In these cases, a multidisciplinary team (MDT) is assigned and general treatment measures may become the main disease management approach. Some oncologists regard front-line chemotherapy as the standard therapeutic regimen for extending progression free survival and overall survival times in patients with metastatic gastric cancer (mGC). New evidence also suggests that secondline salvage chemotherapies confer a survival advantage 
when compared with the best supportive care. But for patients who fail second-line chemotherapy, further effective treatment is needed [4-12]. Molecular targeted therapy may become an effective treatment option for those patients [13-16].

Bevacizumab is a vascular endothelial growth factor (VEGF) inhibitor and widely used in the treatment of tumors, especially lung, breast, kidney, and liver. Its effectiveness in the treatment of colon cancer and other solid tumors has been demonstrated $[17,18]$. Trastuzumab is an effective treatment in patients with Her-2 positive breast and gastric cancers [19-21]. Apatinib is a smallmolecule VEGF receptor (VEGFR) tyrosine kinase inhibitor, similar to vatalanib (PTK787), and has 10 times the binding affinity of vatalanib or sorafenib. The drug has received intellectual property protection in our country as a new kind of oral molecular targeted drug. Investigators in China have carried out a phase I clinical trial and shown that this agent has antitumor activity in Chinese patients with mGC who failed second-line treatment. Safety and efficacy were confirmed by showing the oral drug significantly prolonged progression-free survival and overall survival times $[16,17]$. The Food and Drug Adminstration in China (CFDA) has added apatinib to its list of approved drugs. This phase II, open-label, multicenter, single-arm trial was conducted to assess the safety and efficacy of apatinib as third-line or later treatment in patients with $\mathrm{mGC}$.

\section{MATERIALS AND METHODS}

\section{Patients}

In all, 42 patients (10 male and 32 female; age 35-70 years [median 56]) with histologically confirmed mGC diagnosed at the cancer centers of four hospitals were enrolled from March, 2015 to October, 2015. They had at least one extracranial, not previously irradiated, measurable site of disease according to Response Evaluation Criteria in Solid Tumors (RECIST) 1.1 criteria; had received 1-4 regimens; failed second-line chemotherapy or the last chemotherapy regimen, and had no prior molecular targeted therapy. There were 11 and 21 patients with Eastern Cooperative Oncology Group (ECOG) scores of 1 and 2, respectively, 34 patients with 1 or 2 organ metastases, 8 patients with 2 or more organ metastases. The metastases were located in the liver in 29 patients, lung in 5 patients, and peritoneum in 8 patients.

Six of the 42 patients accepted radiotherapy. Twenty-eight and 14 patients received second-line chemotherapy and subsequent chemotherapy regimens, respectively. Clinical stage I, II, III, and IV mGC was diagnosed in 1, 1, 2, and 39 patients (Table 1).

\section{Study design}

The aim of this open-label, single-arm, multicenter, phase II study was conducted to evaluate the safety and efficacy of apatinib as third-line or later treatment in patients with $\mathrm{mGC}$ and to provide reliable clinical trial data that would inform the design of a large multicenter phase III trial.

\section{Enrollment}

\section{Inclusion criteria}

(1) age $\geq 18$ years, (2) ECOG $\leq 2$, (3) second-line treatment failure, (4) expected survival time more than three months, (5) at least one measurable lesion according to RECIST 1.1 criteria, (6) acceptable liver and kidney function with treatment, and (7) provision of informed consent.

\section{Exclusion criteria}

(1) uncontrolled hypertension $(140 / 90 \mathrm{mmHg}$ or higher), (2) serious coronary heart disease (CHD), (3) treatment with anticoagulants and hemostatic drugs, (4) receiving radiotherapy, and (5) pregnancy or lactation.

\section{Method and dose}

Based on the pharmacokinetic and bioavailability data obtained from the phase I clinical trial, apatinib 850 mg daily was administered 30 minutes postprandially on days 1 through 28 of each 4 -week cycle. The apatinib was supplied by Jiangsu HengRui Medicine Co., Ltd. The dose was reduced to $750 \mathrm{mg}$ per day if patients experienced $3 / 4$ grade adverse events or became intolerant to therapy, further reduced to $500 \mathrm{mg}$ per day if these adverse events persisted, or discontinued if these adverse events persisted even at a dose of $500 \mathrm{mg}$ per day.

\section{Efficacy and adverse events}

Evaluation included physical examination, laboratory tests (CBCs and blood chemistry), and imaging tests (MRI or CT scan of measurable lesions) at baseline and every 2 cycles ( 8 weeks) until disease progression. The objective response rate (ORR) was defined as the rate that patients achieved complete remission (CR) or partial response (PR) by RECIST 1.0 criteria. Disease control rate (DCR) was defined as the rate that patients achieved CR, PR, and stable disease (SD) after at least 8 weeks. Progression-free survival (PFS) was defined as the time to tumor progression or death from any cause. OS was defined as the time to death or the last follow-up.

Adverse events (AEs) were assessed and graded in 
Table 1: patient characteristics

\begin{tabular}{|c|c|c|}
\hline Characteristics & $\mathbf{N}$ & $\%$ \\
\hline \multicolumn{3}{|l|}{ Sex } \\
\hline Male & 32 & 76.2 \\
\hline Female & 10 & 23.8 \\
\hline Median age years & 56 & \\
\hline \multicolumn{3}{|l|}{ ECOG } \\
\hline 1 & 11 & 26.2 \\
\hline 2 & 31 & 73.8 \\
\hline \multicolumn{3}{|l|}{$\begin{array}{l}\text { Prior surgery of primary } \\
\text { tumor }\end{array}$} \\
\hline Yes & 31 & 73.8 \\
\hline No & 11 & 26.2 \\
\hline \multicolumn{3}{|l|}{ Stage } \\
\hline II & 1 & 2.4 \\
\hline III & 2 & 4.8 \\
\hline IV & 39 & 92.8 \\
\hline \multicolumn{3}{|l|}{ No of metastatic sites } \\
\hline$\leq 2$ & 34 & 80.9 \\
\hline$>2$ & 8 & 19.1 \\
\hline \multicolumn{3}{|l|}{$\begin{array}{l}\text { Previous chemotherapy } \\
\text { lines }\end{array}$} \\
\hline 2 & 28 & 66.7 \\
\hline$\geq 3$ & 14 & 33.3 \\
\hline \multicolumn{3}{|l|}{ Metastasis site/organ } \\
\hline Liver & 29 & 69.0 \\
\hline Lung(s) & 5 & 11.9 \\
\hline $\begin{array}{l}\text { Posterior peritoneum } \\
\text { lymph node }\end{array}$ & 8 & 19.1 \\
\hline \multicolumn{3}{|l|}{ Prior radiotherapy } \\
\hline Yes & 6 & 14.3 \\
\hline No & 36 & 85.7 \\
\hline
\end{tabular}

accordance with the Common Terminology Criteria for AEs, version 4.0. The safety evaluation was continued for 28 days after the last dose of apatinib or until recovery to grade 1 or 0 from any acute toxicities associated with apatinib. Blood chemistry and counts were checked weekly, and liver and kidney functions were evaluated every 2 weeks.

\section{The end point}

The primary end point of this study was PFS. The secondary end points included OS, DCR, ORR, and adverse events. The study was terminated under the following circumstances: (1) the efficacy and safety could not be evaluated according to the study protocol; (2) subjects withdrew their informed consent; (3) disease progression; (4) drug toxicity could not be tolerated; and (5) pregnancy.

\section{Follow-up}

Follow-up was by structured record review and telephone interview. Patients were observed until death, loss to follow-up, or end of study. The last time of followup was June 30, 2016.

\section{Statistical analysis}

To estimate sample size, a 7-month accrual period and 10-month follow-up period were assumed. The study was designed with two-sided, $\alpha=0.05,80 \%$ power to detect a null median PFS $(n=40)$. Assuming a $10 \%$ dropout rate, the final accrual number was 44 .

Survival and safety were assessed in patients who received at least one cycle of apatinib. PFS and OS were estimated using Kaplan-Meier analysis. The SPSS 17.0 was used for all statistical analyses. 


\section{RESULTS}

\section{Recruitment and demographics}

The study recruited 42 patients with mGC from 4 hospital cancer centers and was terminated on June 30, 2016. All patients received at least one cycle of oral treatment, and were evaluated after the second and fourth cycles, respectively. After the second cycle assessment, 4 patients withdrew, 3 patients experienced drug intolerance or adverse events, and 2 patients died. Drug dose was reduced to $750 \mathrm{mg}$ per day in 3 patients and to $500 \mathrm{mg}$ per day in one. A total of 33 patients received a third cycle. After four cycles, one patient dropped out of the study, 5 patients experienced drug intolerance or adverse events, and 10 patients died. Drug dose was reduced after the first cycle by 6 patients, the second cycle by 3 patients, and 24 patients completed all four cycles (Figure 1).

\section{Efficacy}

At the date of last follow-up (June 30, 2016), all patients had undergone 1-4 cycles of chemotherapy, and

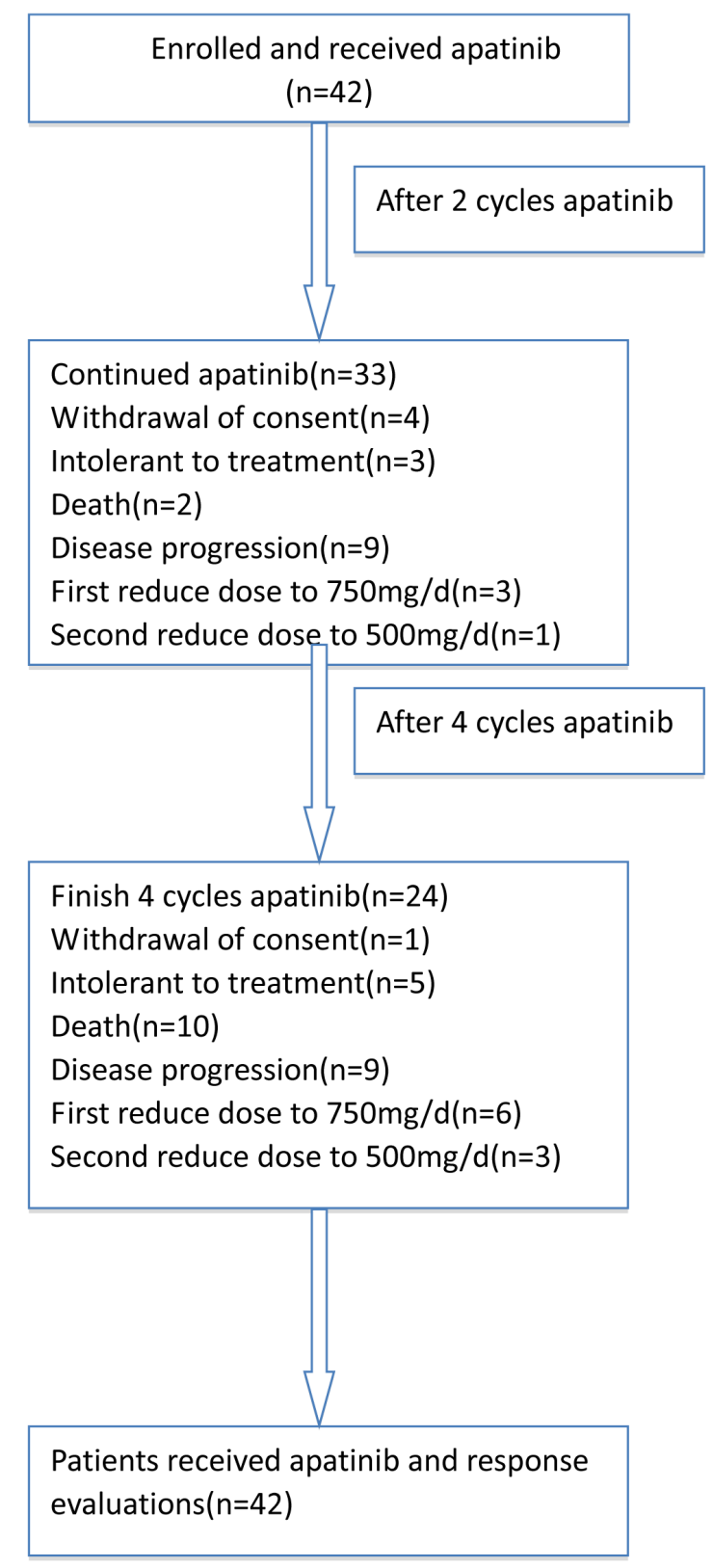

Figure 1: CONSORT Diagram, enrollment and outcomes 
Table2: Disease response after treatment

\begin{tabular}{llllll}
\hline & CR & PR & SD & PD & DCR $\%$ \\
\hline After 2 cycles apatinib & 0 & 4 & 29 & 9 & $33(78.57 \%)$ \\
& & & & & \\
After 4 cycles apatinib & 1 & 7 & 16 & 9 & $24(57.14 \%)$ \\
\hline
\end{tabular}

Table 3: Adverse Event based on CTCAE 4.0

\begin{tabular}{lcccccc}
\hline Adverse Event & \multicolumn{6}{c}{ Adverse Event Grade } \\
\cline { 2 - 7 } & 0 & 1 & 2 & 3 & 4 & Total (n,\%) \\
\hline Hypertension & 27 & 8 & 4 & 3 & 0 & $15(35.71)$ \\
Hand-foot syndrome & 25 & 11 & 5 & 1 & 0 & $17(40.48)$ \\
Fatigue & 36 & 4 & 2 & 0 & 0 & $6(14.29)$ \\
Diarrhea & 35 & 4 & 2 & 1 & 0 & $7(16.67)$ \\
Pain & 35 & 3 & 3 & 1 & 0 & $7(16.67)$ \\
Proteinuria & 37 & 5 & 0 & 0 & 0 & $5(11.90)$ \\
Nausea & 37 & 2 & 1 & 1 & 0 & $5(11.90)$ \\
Vomiting & 30 & 8 & 4 & 0 & 0 & $12(28.57)$ \\
Anemia & 33 & 7 & 2 & 0 & 0 & $9(21.43)$ \\
Leukopenia & 27 & 7 & 8 & 0 & 0 & $15(35.71)$ \\
Aminotransferase & 23 & 9 & 7 & 3 & 0 & $19(45.24)$ \\
$\quad$ increased & & & & & & \\
$\quad$ Thrombocytopenia & 32 & 6 & 3 & 1 & 0 & $10(23.81)$ \\
Neutropenia & 30 & 7 & 5 & 0 & 0 & $12(28.57)$ \\
Dizziness & 39 & 3 & 0 & 0 & 0 & $3(7.14)$ \\
\hline
\end{tabular}

achieved a median PFS time of 4.0 months $(95 \%$ CI, 2.85$5.15)$ and median OS time of 4.50 months $(95 \% \mathrm{CI}, 4.03-$ 4.97). After 2 cycles, there were 9, 29, and 9 patients who experienced PR, SD, and disease progression (PD), and the DCR and ORR were $78.57 \%$ and $9.52 \%$, respectively. After 4 cycles, 1, 7, 16, and 9 patients had PR, SD, and PD, for a DCR of $57.14 \%$ and ORR of $19.05 \%$ (Table 2).

\section{Safety}

All patients were evaluated for drug toxicity and adverse events, which occurred in 38 patients to varying degree. These included secondary hypertension, hand-foot syndrome, fatigue, anemia, leukopenia, thrombocytopenia, diarrhea, vomiting, changes in the skin and mucous membranes, and so on. Toxicities were generally well tolerated. Secondary hypertension, elevated aminotransferase, and hand-foot syndrome were the main adverse reactions with incidence rates of $35.71 \%, 45.24 \%$, and $40.48 \%$, respectively. The secondary hypertension and elevated aminotransferase were the main grade $3 / 4$ adverse events, each occurring in 3 patients and at an incidence rate of $7.14 \%$. The total adverse event rate was $20.0 \%$ (Table 3). 


\section{DISCUSSION}

Gastric cancer is among the most common malignancies in East Asian countries. Recurrence and metastasis are the biggest obstacles to successful treatment of gastric cancer. Eating habits are one of the important reasons. Medical conditions and diagnosis time are related to mortality. Many patients with gastric cancer already have metastases by the time they visit the hospital and so are no longer considered good candidates for surgery. The standard first-line treatment for $\mathrm{mGC}$ can slow disease progression and improve overall survival. New clinical evidence shows that some patients can benefit from secondary rescue chemotherapy [4-8, 22]. But for those who fail rescue treatment, there are a lack of effective treatments. Molecular targeted drugs bring new hope for those patients. Trastuzumab has been confirmed to be an effective treatment in patients with Her-2 gene positive breast cancer and gastric cancer [19, 20, 23]. However, dealing with Her-2 negative disease remains a clinical challenge. Apatinib is a small-molecule VEGFR tyrosine kinase inhibitor, similar to vatalanib (PTK787), but with a binding affinity 10 times that of vatalanib or sorafenib. It is a new oral molecular targeted drug that has been approved by the CFDA in China. This study investigated the efficacy of apatinib in patients with gastric cancer who experienced progression after receiving two or more lines of chemotherapy. It is an open-label, single-arm, multicenter, phase II study. All 42 patients enrolled in the study were treated with apatinib and evaluated. After taking one cycle of $850 \mathrm{mg}$ per day for 28 days [24, 25], the median PFS and OS times were 4.0 months (95\% CI, 2.85-5.15) and 4.50 months (95\% CI, 4.03-4.97). Similarly, Li [25] reported that once daily $850 \mathrm{mg}$ led to median PFS and OS times of 3.67 months and 4.83 months. In this study, the DCR and ORR were $78.57 \%$ and $9.52 \%$ after 2 cycles and $57.14 \%$ and $19.05 \%$ after 4 cycles. Adverse events (AEs) of varying degree occurred in 38 patients, including secondary hypertension, hand-foot syndrome, fatigue, anemia, leukopenia,

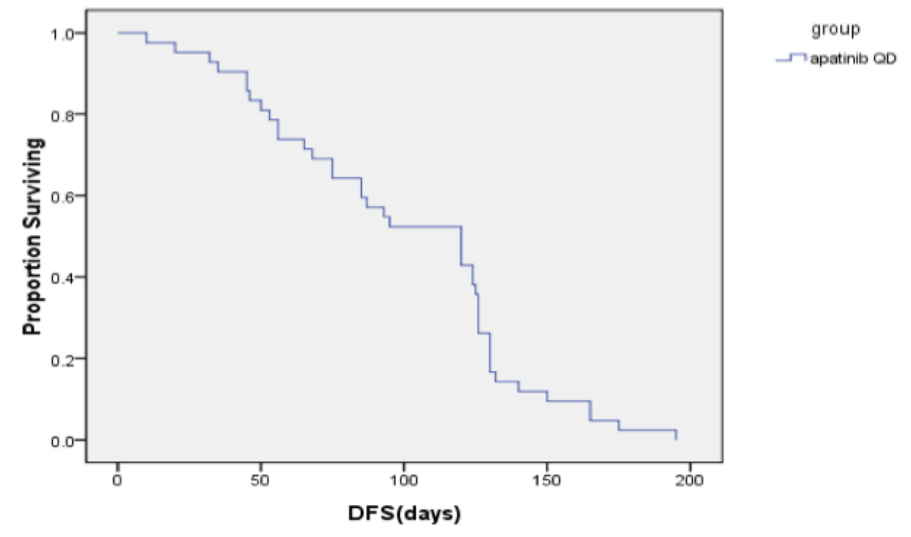

Figure 2: Progression survival table

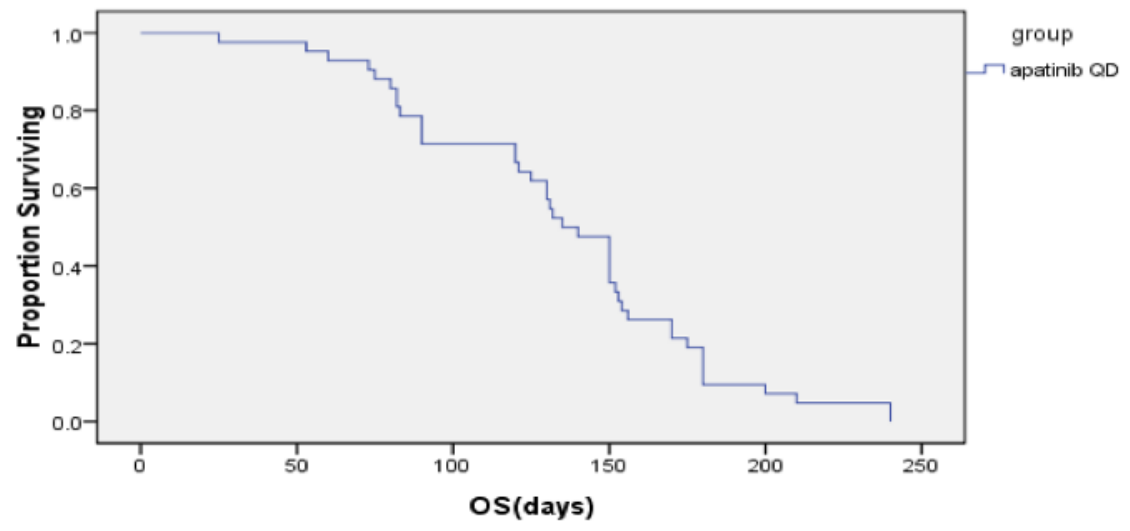

Figure 3: Overall survival. 
thrombocytopenia, diarrhea, vomiting, changes in the skin and mucous membranes, and so on. The leading AEs (with incidence rates) were secondary hypertension (35.71\%), elevated aminotransferase (45.24\%), and hand-foot syndrome $(40.48 \%)$. Grade 3 to 4 AEs were secondary hypertension $(n=3,7.14 \%)$ and elevated aminotransferase $(n=3,7.14 \%)$. The total AE rate was $20.0 \%$. Drug dose was reduced in 9 patients because of grade 3 to 4 AEs, and treatment was terminated in 8 patients because of intolerance to AEs. Li [25] reported secondary hypertension and hand-foot syndrome as the main AEs and hematological toxicities of rarely more than grade 3 . Hu [26] reported an incidence of grade 3 to 4 AEs (secondary hypertension and hand-foot syndrome) of $20.5 \%$ and $10.3 \%$, respectively, in patients treated with apatinib for triple negative breast cancer. The incidence of AEs was higher in our study than in other studies, possibly because our mGC patients were in poorer condition and less tolerant.

In summary, this study was an open-label, singlearm, multicenter, phase II study of the safety and efficacy of apatinib (850 mg once daily). Patients given apatinib had high incidence of grade 3 to 4 AEs, which can be reduced by decreasing the dose.

\section{ACKNOWLEDGMENTS}

The authors wish to thank all of the individuals who assisted with this study.

\section{CONFLICTS OF INTEREST}

The authors declare that they have no conflicts of interest.

\section{FUNDING}

This study was supported by a grant from the Science and Technology Committee of Jiangxi Province, China (no.: 20161BBG700100).

\section{REFERENCES}

1. Jemal A, Bray F, Center MM, Ferlay J, Ward E, Forman D. Global cancer statistics. CA Cancer J Clin. 2011;2:69-90.

2. Torre LA, Bray F, Siegel RL, Ferlay J, Lortet-Tieulent J, Jemal A. Global cancer statistics, 2012. CA Cancer J Clin. 2015;2: 87-108

3. Liu X, Qiu H, Liu J, Chen S, Xu D, Li W, Zhan Y, Li Y, Chen Y, Zhou Z, Sun X. Combined preoperative concentrations of CEA, CA 19-9, and 72-4 for predicting outcomes in patients with gastric cancer after curative resection. Oncotarget. 2016; 7:35446-53. https://doi. org/10.18632/oncotarget.9060

4. Lindsey A. Torre MSPH and Ann M.Cancer statistics for
Asian Americans, Native Hawaiians, and Pacific Islanders, 2016: Converging incidence in males and females. CA Cancer J Clin. 2016;3:182-202.

5. Kang JH, Lee SI, Lim DH, Park KW, Oh SY, Kwon HC, Hwang IG, Lee SC, Nam E, Shin DB, Lee J, Park JO, Park YS, et al. Salvage chemotherapy for pretreated gastric cancer: a randomized phase III trial comparing chemotherapy plus best supportive care with best supportive care alone. J Clin Oncol. 2012; 30:1513-18.

6. Hawkes E, Okines AF, Papamichael D, Rao S, Ashley S, Charalambous H, Koukouma A, Chau I, Cunningham D. Docetaxel and irinotecan as second-line therapy for advanced oesophagogastric cancer. Eur J Cancer. 2011; 47:1146-51.

7. Fuchs CS, Tomasek J, Yong CJ, Dumitru F, Passalacqua R, Goswami C, Safran H, Dos Santos LV, Aprile G, Ferry DR, Melichar B, Tehfe M, Topuzov E, et al, and REGARD Trial Investigators. Ramucirumab monotherapy for previously treated advanced gastric or gastro-oesophageal junction adenocarcinoma (REGARD): an international, randomised, multicentre, placebo-controlled, phase 3 trial. Lancet. 2014; 383:31-39.

8. Fazio N, Biffi R, Maibach R, Hayoz S, Thierstein S, Brauchli P, Bernhard J, Stupp R, Andreoni B, Renne G, Crosta C, Morant R, Chiappa A, et al, and Swiss Group for Clinical Cancer Research SAKK, and European Institute of Oncology, Milan, Italy. Preoperative versus postoperative docetaxel-cisplatin-fluorouracil (TCF) chemotherapy in locally advanced resectable gastric carcinoma: 10-year follow-up of the SAKK 43/99 phase III trial. Ann Oncol. 2016; 27:668-73.

9. Hou J, Zhou J. WWC3 downregulation correlates with poor prognosis and inhibition of Hippo signaling in human gastric cancer. Onco Targets Ther. 2017; 10:2931-42.

10. Lote H, Spiteri I, Ermini L, Vatsiou A, Roy A, McDonald A, Maka N, Balsitis M, Bose N, Simbolo M, Mafficini A, Lampis A, Hahne JC, et al. Carbon dating cancer: defining the chronology of metastatic progression in colorectal cancer. Ann Oncol. 2017; 6:1243-49.

11. Waddell T, Chau I, Cunningham D, Gonzalez D, Okines AF, Okines C, Wotherspoon A, Saffery C, Middleton G, Wadsley J, Ferry D, Mansoor W, Crosby T, et al. Epirubicin, oxaliplatin, and capecitabine with or without panitumumab for patients with previously untreated advanced oesophagogastric cancer (REAL3): a randomised, open-label phase 3 trial. Lancet Oncol. 2013; 14:481-89.

12. Hierro C, Alsina M, Sánchez M, Serra V, Rodon J, Tabernero J. Targeting the fibroblast growth factor receptor 2 in gastric cancer: promise or pitfall? Ann Oncol. 2017; 6:1207-16.

13. Geng R, Li J. Apatinib for the treatment of gastric cancer. Expert Opin Pharmacother. 2015; 16:117-22.

14. Wilke H, Muro K, Van Cutsem E, Oh SC, Bodoky G, Shimada Y, Hironaka S, Sugimoto N, Lipatov O, Kim TY, Cunningham D, Rougier P, Komatsu Y, et al, and 
RAINBOW Study Group. Ramucirumab plus paclitaxel versus placebo plus paclitaxel in patients with previously treated advanced gastric or gastro-oesophageal junction adenocarcinoma (RAINBOW): a double-blind, randomised phase 3 trial. Lancet Oncol. 2014; 15:1224-35.

15. Ford HE, Marshall A, Bridgewater JA, Janowitz T, Coxon FY, Wadsley J, Mansoor W, Fyfe D, Madhusudan S, Middleton GW, Swinson D, Falk S, Chau I, et al, and COUGAR-02 Investigators. Docetaxel versus active symptom control for refractory oesophagogastric adenocarcinoma (COUGAR-02): an open-label, phase 3 randomised controlled trial. Lancet Oncol. 2014; 15:78-86.

16. Hecht JR, Bang YJ, Qin SK, Chung HC, Xu JM, Park JO, Jeziorski K, Shparyk Y, Hoff PM, Sobrero A, Salman P, Li J, Protsenko SA, et al. Lapatinib in combination with capecitabine plus oxaliplatin in human epidermal growth factor receptor 2-positive advanced or metastatic gastric, esophageal, or gastroesophageal adenocarcinoma: TRIO013/LOGiC-a randomized phase III trial. J Clin Oncol. 2016; 34:443-51.

17. Ohtsu A, Shah MA, Van Cutsem E, Rha SY, Sawaki A, Park SR, Lim HY, Yamada Y, Wu J, Langer B, Starnawski M, Kang YK. Bevacizumab in combination with chemotherapy as first-line therapy in advanced gastric cancer: a randomized, double-blind, placebo-controlled phase III study. J Clin Oncol. 2011; 29:3968-76.

18. Schwartzberg LS, Tauer KW, Hermann RC, Makari-Judson G, Isaacs C, Beck JT, Kaklamani V, Stepanski EJ, Rugo HS, Wang W, Bell-McGuinn K, Kirshner JJ, Eisenberg $\mathrm{P}$, et al. Sorafenib or placebo with either gemcitabine or capecitabine in patients with HER-2-negative advanced breast cancer that progressed during or after bevacizumab. Clin Cancer Res. 2013; 19:2745-54.

19. Bang YJ, Van Cutsem E, Feyereislova A, Chung HC, Shen L, Sawaki A, Lordick F, Ohtsu A, Omuro Y, Satoh T, Aprile G, Kulikov E, Hill J, et al, and ToGA Trial Investigators. Trastuzumab in combination with chemotherapy versus chemotherapy alone for treatment of HER2-positive advanced gastric or gastro-oesophageal junction cancer (ToGA): a phase 3, open-label, randomised controlled trial. Lancet. 2010; 376:687-97.

20. Satoh T, Xu RH, Chung HC, Sun GP, Doi T, Xu JM, Tsuji A, Omuro Y, Li J, Wang JW, Miwa H, Qin SK, Chung IJ, et al. Lapatinib plus paclitaxel versus paclitaxel alone in the second-line treatment of HER2-amplified advanced gastric cancer in Asian populations: TyTAN-a randomized, phase III study. J Clin Oncol. 2014; 32:2039-49.

21. Bekaii-Saab, El-Rayes B. Identifying and targeting cancer stem cells in the treatment of gastric cancer. Cancer. 2017;8:1303-1312.

22. Mao Y, Liu R, Zhou H, Yin S, Zhao Q, Ding X, Wang H. Transcriptome analysis of miRNA-lncRNA-mRNA interactions in the malignant transformation process of gastric cancer initiation. Cancer Gene Ther. 2017; 24:26775.
23. Liu P, Zhu Y, Liu L. Elevated serum CA72-4 levels predict poor prognosis in pancreatic adenocarcinoma after intensity-modulated radiation therapy. Oncotarget. 2015; 6:9592-99. https://doi.org/10.18632/oncotarget.3562

24. Tian S, Quan H, Xie C, Guo H, Lü F, Xu Y, Li J, Lou L. YN968D1 is a novel and selective inhibitor of vascular endothelial growth factor receptor-2 tyrosine kinase with potent activity in vitro and in vivo. Cancer Sci. 2011; 102:1374-80.

25. Li J, Qin S, Xu J, Guo W, Xiong J, Bai Y, Sun G, Yang Y, Wang L, Xu N, Cheng Y, Wang Z, Zheng L, et al. Apatinib for chemotherapy-refractory advanced metastatic gastric cancer: results from a randomized, placebo-controlled, parallel-arm, phase II trial. J Clin Oncol. 2013; 31:3219-25.

26. Hu X, Cao J, Hu W, Wu C, Pan Y, Cai L, Tong Z, Wang S, Li J, Wang Z, Wang B, Chen X, Yu H. Multicenter phase II study of apatinib in non-triple-negative metastatic breast cancer. BMC Cancer. 2014; 14:820-24. 